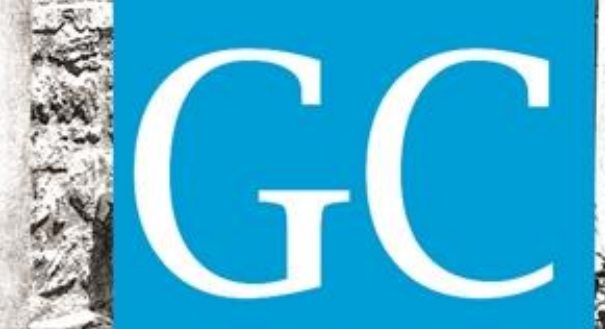

Revista Nacional de Gerenciamento de Cidades

\title{
Infraestrutura verde na cidade contemporânea
}

Green infrastructure in the contemporary city

Infraestructura verde en la ciudad contemporánea

Sandra Medina Benini

Doutora em Arquitetura e Urbanismo Docente do Centro Universitário de Várzea Grande - UNIVAG arquiteta.benini@gmail.com

\section{Jeane Aparecida Rombi de Godoy Rosin}

Doutora em Arquitetura e Urbanismo Docente do Centro Universitário de Várzea Grande - UNIVAG urbanista.jeane@gmail.com 


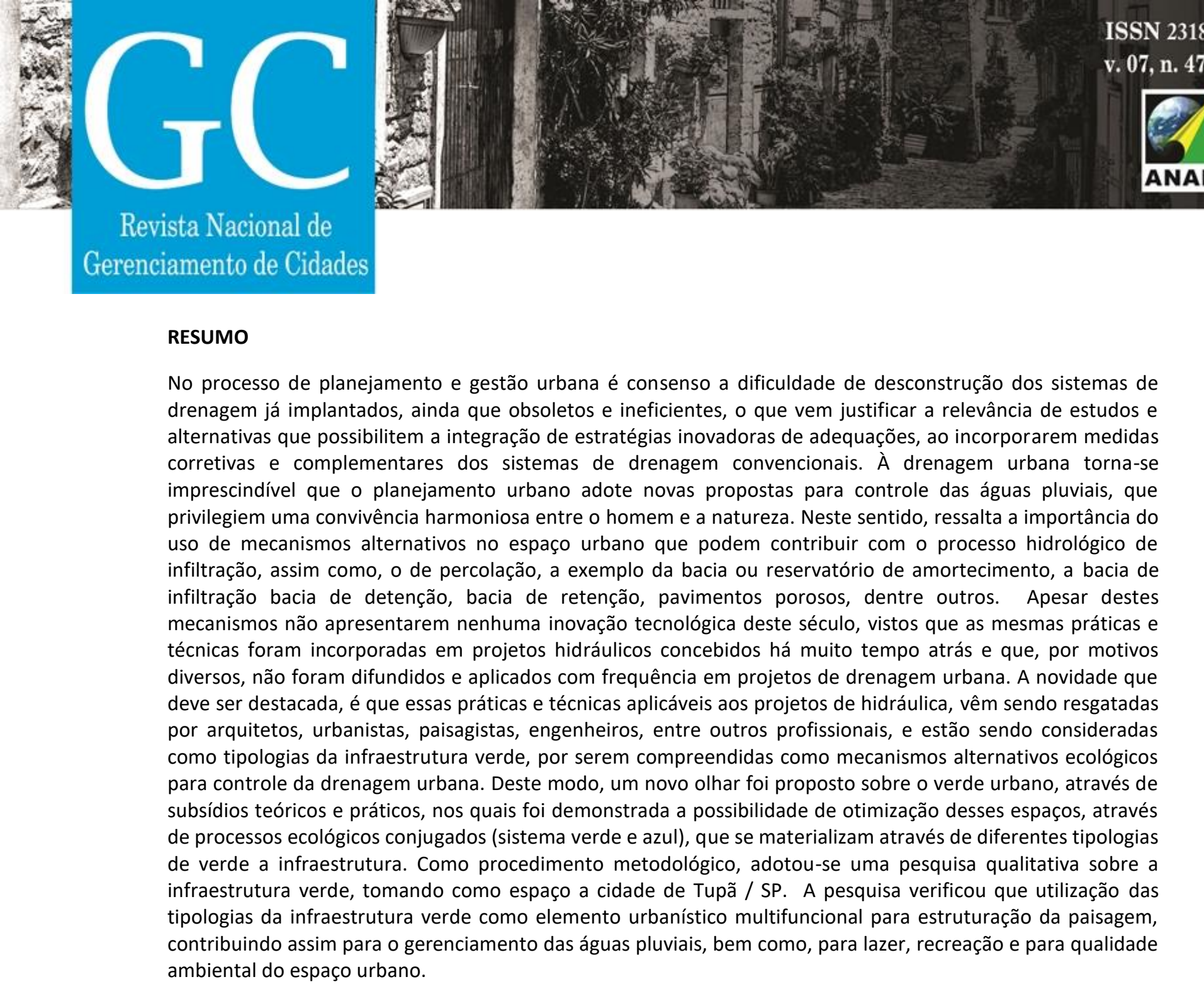

Palavras-chave: Cidade Contemporânea. Meio Ambiente. Gestão.

\section{SUMMARY}

In the urban planning and management process, the difficulty of deconstructing drainage systems already implemented, although obsolete and inefficient, is consensus, which justifies the relevance of studies and alternatives that enable the integration of innovative adaptation strategies, by incorporating corrective measures and complementary to conventional drainage systems. For urban drainage, it is essential that urban planning adopts new proposals for rainwater control, which favor a harmonious coexistence between man and nature. In this sense, it emphasizes the importance of the use of alternative mechanisms in the urban space that can contribute to the hydrological infiltration process, as well as the percolation, such as the dam catchment or reservoir, the detention basin, retention, porous floors, among others. Although these mechanisms do not present any technological innovation of this century, since the same practices and techniques were incorporated in hydraulic projects conceived long ago and, for various reasons, were not widespread and often applied in urban drainage projects. The novelty that should be highlighted is that these practices and techniques applicable to hydraulics projects have been rescued by architects, city planners, landscape designers, engineers, among other professionals, and are being considered as typologies of green infrastructure, as they are understood as mechanisms. Ecological alternatives for urban drainage control. Thus, a new look was proposed on the urban green, through theoretical and practical subsidies, which demonstrated the possibility of optimizing these spaces, through conjugated ecological processes (green and blue system), which materialize through different typologies. From green to infrastructure. As a methodological procedure, a qualitative research on the green infrastructure was adopted, taking as space the city of Tupã / SP. The research verified that the use of green infrastructure typologies as a multifunctional urban element for 


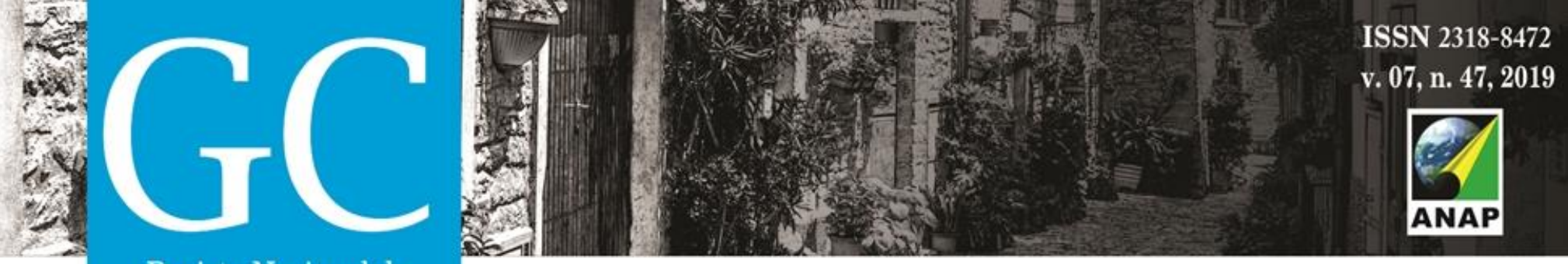

\section{Revista Nacional de}

\section{Introdução}

No processo de planejamento e gestão urbana é consenso a dificuldade de desconstrução dos sistemas de drenagem já implantados, ainda que obsoletos e ineficientes, o que vem justificar a relevância de estudos e alternativas que possibilitem a integração de estratégias inovadoras de adequações, ao incorporarem medidas corretivas e complementares dos sistemas de drenagem convencionais. A partir desses pressupostos, o planejamento urbano e o desenho ambiental possibilitam a conjugação de conceitos e técnicas ao incorporar princípios e técnicas com capacidade de respostas adequadas aos efeitos decorrentes das deficiências do sistema de drenagem, que vêm produzindo inundações em diversas cidades do país.

À drenagem urbana torna-se imprescindível que o planejamento urbano adote novas propostas para controle das águas pluviais, que privilegiem uma convivência harmoniosa entre o homem e a natureza. Neste sentido, diversos autores, dentre eles, Botelho (2011, p. 93) defendem a ideia de que é "preciso desenvolver novas formas de ocupação, novos materiais, novas técnicas, novas leis, estabelecendo novas relações de uso do espaço urbano", notadamente ao considerar que "a ideia de 'desconstruir' cidades é inconcebível e ilusória, pois não podemos deixar de construir moradias, asfaltar ruas, erguer centros empresariais etc.".

Neste sentido, ressalta a importância do uso de mecanismos alternativos no espaço urbano que podem contribuir com o processo hidrológico de infiltração, assim como, o de percolação, a exemplo da bacia ou reservatório de amortecimento, a bacia de infiltração bacia de detenção, bacia de retenção, pavimentos porosos, dentre outros.

Apesar destes mecanismos não apresentarem nenhuma inovação tecnológica deste século, vistos que as mesmas práticas e técnicas foram incorporadas em projetos hidráulicos concebidos há muito tempo atrás e que, por motivos diversos, não foram difundidos e aplicados com frequência em projetos de drenagem urbana. A novidade que deve ser destacada, é que essas práticas e técnicas aplicáveis aos projetos de hidráulica, vêm sendo resgatadas por arquitetos, urbanistas, paisagistas, engenheiros, entre outros profissionais, e estão sendo consideradas como tipologias da infraestrutura verde, por serem compreendidas como mecanismos alternativos ecológicos para controle da drenagem urbana.

Segundo Herzog (2013, p. 111), a infraestrutrura verde, também chamada de "infraestrutura ecológica, é um conceito que tem evoluído rapidamente e se tornado mais abrangente nos últimos anos" e se fundamenta nos "conhecimentos da ecologia da paisagem e da ecologia urbana. Compreende a cidade como um sistema socioecológico, por meio de uma visão holística, sistêmica". Ferreira e Machado (2010, p. 81) explicam que infraestrutura verde quando utilizada em projetos urbanos, insere, conserva ou recupera a "estrutura ecológica" do local da intervenção, por possibilitar a integração do "sistema azul" (circulação da água) com o "sistema verde" (produção de biomassa). 


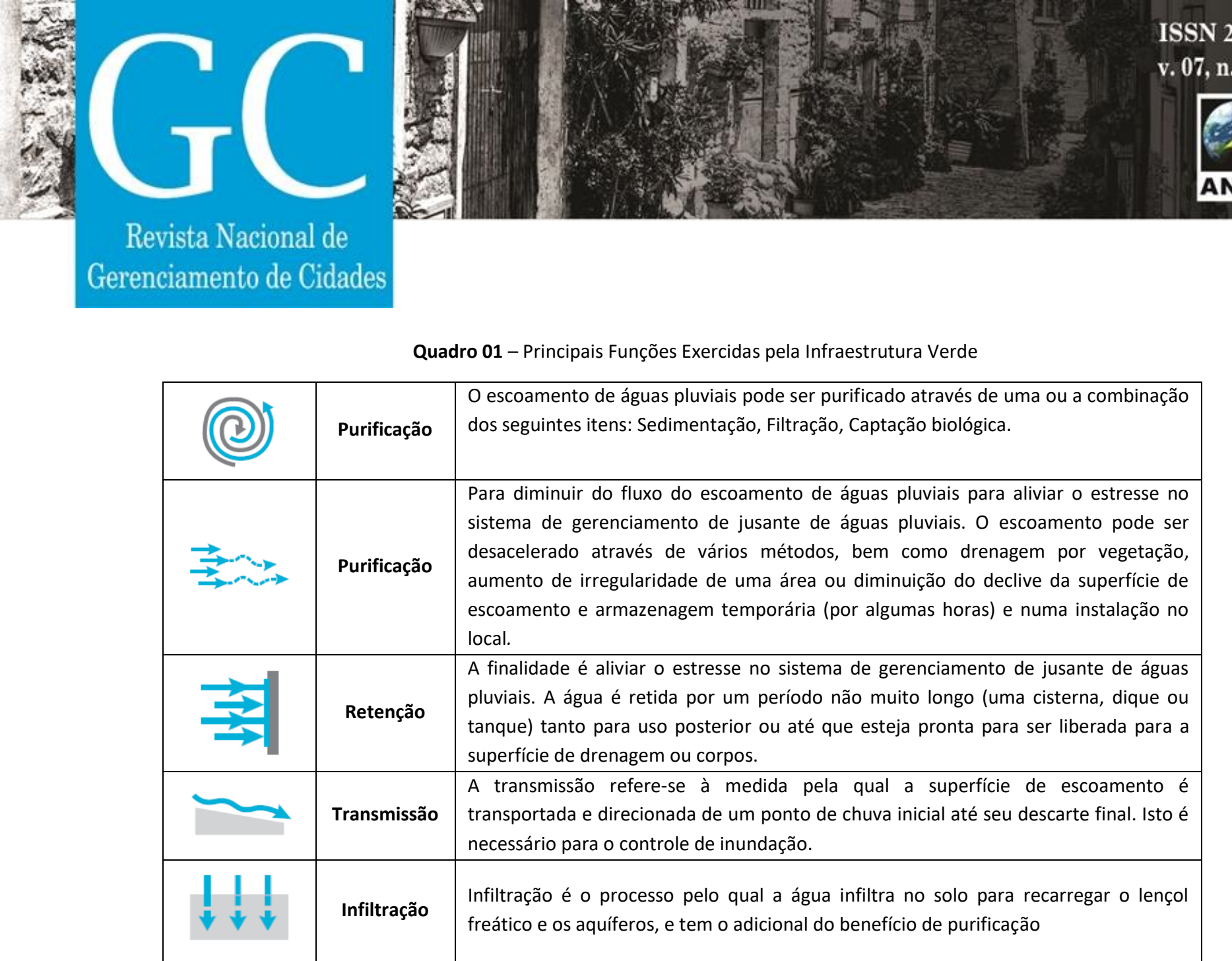

Fonte: SINGAPORE, 2011, p. 30-31 - Tradução Nossa

Entretanto, deve ser destacado que as funções da infraestrutura verde terão maior eficácia se houver interconexão com uma rede natural de espaços verdes, conservando os serviços ecossistêmicos.

Neste sentido, antes de qualquer intervenção urbana, devem ser considerados os problemas e potencialidades ambientais do local, tendo em vista a incorporação dos princípios e técnicas da infraestrutura verde, a qual permite identificar os espaços de maior fragilidade - ambientalmente mais sensíveis, portanto prioritários para o tratamento e alcance das possibilidades oferecidas.

Desse modo, torna-se necessário, a revisão dos modelos convencionais, bem como a adoção de técnicas alternativas, pautadas por princípios ambientais que considerem a possibilidade de construção da sustentabilidade urbana.

\section{Benefícios da Infraestrutura Verde}

Benedict e McMahon (2002a) destacam que a infraestrutura verde promove a proteção e ajuda a restaurar os ecossistemas naturalmente.

Green infrastructure systems help protect and restore naturally functioning ecosystems by providing a framework for future development that fosters a diversity of ecological, social, and economic benefits. These include enriched habitat and biodiversity; maintenance of natural landscape processes; cleaner air and 


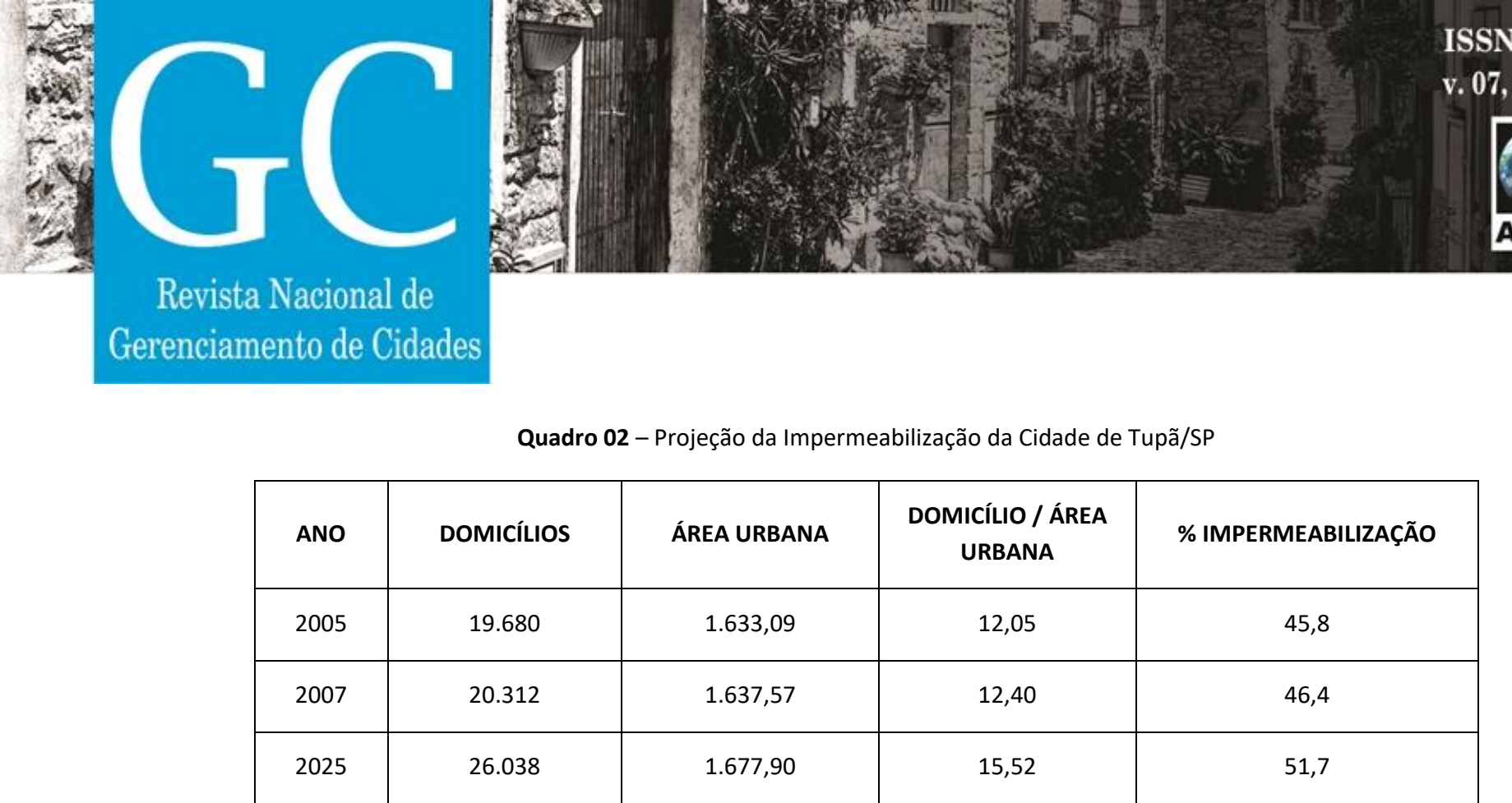

Fonte: Fundação Centro Tecnológico de Hidráulica, 2008a. Adaptado por Sandra Medina Benini, 2009.

Conforme observado no Quadro 02 - Projeção da Impermeabilização da Cidade de Tupã, o percentual de impermeabilização encontrado, deverá aumentar 5,9\% desde 2007 até 2025.

Em razão da magnitude dos impactos produzidos, durante as últimas décadas foi dado início a um processo de revisão dos procedimentos técnicos empregados no sistema de drenagem urbana, resultando em significativas reformulações conceituais e práticas. Foi essa nova visão que norteou a elaboração de Estudos de Macrodrenagem Urbana da Estância Turística de Tupã (Plano de Macrodrenagem - composto pelo Plano de Ação Imediata e o Plano de Ação Continuada).

Segundo a Fundação Centro Tecnológico de Hidráulica (2008e, p. 94), a execução do "Plano de Macrodrenagem em sua essência" (Plano de Ação Imediata e o Plano de Ação Continuada) tem por objetivo melhorar a qualidade de vida da população tupãense "no cenário atual", por meio de "investimento da ordem de R\$ 69.000.000,00 até 2027".

Em 2010, foi viabilizada a assinatura do convênio com o Governo Federal por meio do Programa de Aceleração do Crescimento (PAC) Saneamento, marcando o início de execução das obras prevista no Plano de Macrodrenagem. Nessa primeira etapa (vinculada ao Plano de Ação Imediata) as obras foram orçadas em 24,5 milhões de reais, sendo:

- 10,5 milhões a fundo perdido;

- 12,8 milhões serão liberados através de financiamento já aprovado pela Caixa Econômica Federal;

- 1,2 milhão será a contrapartida do Município.

Todavia, deve-se destacar que uma vez vinculados os recursos públicos ao Plano Macrodrenagem, no caso específico em estudo, necessariamente, o administrador público poderá ser responsabilizado a qualquer tempo, por ações ou omissões que venham comprometer a execução desse plano.

Neste sentido, a Fundação Centro Tecnológico de Hidráulica (2008e) ressalta que

[...] as medidas estruturais devem e virão acompanhadas de outras medidas denominadas, compensatórias e não-estruturais. Estas, na grande maioria, de caráter institucional serão parte integrante do PAC e, como tal, deverão ser 
